J. Clin. Chem. Clin. Biochem.

Vol. 19, 1981, pp. 295-299

\title{
Wachstum von Calciumoxalatkristallen in vitro: Einfache Methode zur Messung der Hemmwirkung menschlichen Urins
}

\author{
Von $B$. Hess und $U$. Binswanger
}

Departement für Innere Medizin, Nephrologische Station, Universitätsspital Zürich

(Eingegangen am 24. Juni 1980/3. Februar 1981)

Zusammenfassung: Es wird eine technisch einfache und reproduzierbare Methode zur Erfassung des CalciumoxalatMonohydrat-Kristallwachstums in vitro vorgestellt. In einem bezüglich Calcium- und Oxalat-Ionenkonzentrationen metastabilen System wird nach Zugabe einer konstanten Menge von Calciumoxalat-"seed-crystals" das Kristallwachstum anhand der Abnahme freier Calciumionen im Zeitverlauf verfolgt. Die Untersuchungen beschränken sich auf die Bestimmung der globalen Hemmkraft im Versuchsansatz nach Zugabe von verschiedenen Inhibitoren sowie Spontanurinen, während das Problem der Ubersättigung an kristallbildenden Ionen durch Verwendung niedriger Konzentrationen ausgeschlossen wird. Von den bekannten getesteten Inhibitoren Ethan-1-hydroxy-1,1-bisphosphonat, Heparin und Chondroitinsulfat $C$ wirkt Ethan-1-hydroxy-1,1-bisphosphonat am stärksten kristallwachstumshemmend. Die Hemmkraft menschlichen Urins wird durch Zugabe von Spontanurin $(30 \mathrm{ml} / \mathrm{l})$ nüchterner und stark hydrierter Probanden geprüft.

\section{Growth of calcium oxalate crystals in vitro:}

A simple method for measurement of the inhibitory action of human urine

Summary: A technically simple and reproducible method is described for the determination of the growth of crystals of calcium oxalate monohydrate in vitro. In a system made metastable with respect to calcium and oxalate ion concentrations, the time course of crystal growth was measured by the decrease of free calcium ions, following the addition of a fixed quantity of calcium oxalate seed crystals. Inhibition of crystal growth caused by the addition of various inhibitors or urine was determined, and the possibility of supersaturation of crystal-forming ions was excluded by using low concentrations. The known inhibitors, ethane 1-hydroxy-1,1-bisphosphonate, heparin and chondroitin sulphate $C$ were tested, and ethane 1-hydroxy-1,1-bisphosphonate showed the strongest inhibition of crystal growth. The inhibitory action of human urine was tested by addition of urine $(30 \mathrm{ml} / \mathrm{l})$ from resting and strongly hydrated probands.

\section{Einführựng}

Nach Williams (1) enthalten $2 / 3$ aller in den USA vorkommenden Nierensteine Calciumoxalat, sei es als alleinige Komponente oder gemischt mit Calciumphosphat (Hydroxylapatit). Wie beim Ausfallen anderer Konkremente aus dem Harn spielen auch bei der Entstehung von Calciumoxalatsteinen die initiale Nukleation (Kristallbildung) sowie Wachstum und Aggregation der entstandenen Mikrokristalle eine entscheidende Rolle $(2,3)$.

Die spontane Nukleation erfolgt bei starker Übersättigung des Urins an kristallbildenden Ionen. In nur schwach übersättigten Lösungen erfolgt eine Kristallbildung jedoch nur in Anwesenheit des auszufällenden Salzes oder eines in seiner kristallinen Struktur ähnlichen Nukleators (homogene und heterogene Nukleation). Der früher ebenfalls diskutierten Nukleation um eine organische Matrix wird heute nur noch geringe Bedeutung beigemessen $(2,3)$.

Kristallwachstum und Kristallaggregation sollen hauptșächlich von 2 Faktoren abhängig sein (4): Erstens wiederum vom Grad der Urinübersättigung, zweitens aber vom Gehalt des Urins an Inhibitoren. Die Suche nach solchen Substanzen im menschlichen Urin gewann zusehends an Bedeutung, seit Thomas \& Howard 1959 entdeckten (5), daß Urin normaler Probanden den Knorpel rachitischer Ratten nicht zu mineralisieren vermag, wohl aber Urin ausgewählter Nierensteinpatienten. Robertson \& Peacock (6) gelang der Nachweis, daß menschlicher Urin in vitro Wachstum und Aggregation von Calciumoxalatkristallen zu hemmen vermag. 
Verschiedene Inhibitoren wurden identifiziert und in vitro getestet. Pyrophosphat hemmt neben dem Calciumphosphat-Kristallwachstum (7) sehr stark Wachstum und Aggregation von Calciumoxalatkristallen $(8,9)$. Citrat hemmt wohl das Wachstum von Calciumphosphatkristallen, hat aber auf das Calciumoxalat-Kristallwachstum nur einen geringen inhibitorischen Effekt $(7,8)$. Magnesiumionen schließlich vermögen in physiologischen Konzentrationen ausschließlich das Calciumphosphat-Kristallwachstum zu hemmen $(7,8)$. Ethan-hydroxy-1,1-bisphosphonat ${ }^{1}$ ) wurde sowohl in vitro als auch in vivo als potenter Inhibitor der Calciumoxalat-Aggregation beschrieben $(2,10,11)$.

Immer wieder werden auch Makromoleküle wie die sauren Mucopolysaccharide als wesentlichste Hemmer der Calciumoxalat-Aggregation diskutiert. In vitro erwiesen sich Heparin und Chondroitinsulfat als wirksame Inhibitoren (9). Die Isolation saurer Mucopolysaccharide im Urin ist methodisch problematisch.

Die vorliegende Arbeit hat zum Zweck, eine einfache und reproduzierbare Methode zur Durchführung von Calciumoxalat-Monohydrat-Kristallwachstumsexperimenten in vitro darzulegen. Dabei interessiert ausschließlich die Hemmung des Kristallwachstums, währenddem das Problem der Übersättigung an kristallbildenden Ionen durch Verwendung niedriger Ionenkonzentrationen ausgeschlossen wird. Die Hemmkraft bekannter Inhibitoren und verdünnter Spontanurine von Normalpersonen wird gemessen.

\section{Material und Methodik}

Die Calciumoxalat-Anlagerung an "seed crystals" (Calciumoxalat-Monohydrat) in einer bezüglich $\mathrm{Ca}^{++}$- und Oxalat-Ionen metastabilen Lösung (1:1-Relation von $\left[\mathrm{Ca}^{++}\right]$und $\left.\left[(\mathrm{COO})_{2}^{2-}\right]\right)$ wird nach Zusatz verdünnten Urins $(30 \mathrm{ml} / \mathrm{l})$ und CalciumoxalatKristallsuspension ( $5 \mathrm{ml} / \mathrm{l}$ ) anhand der Abnahme freier Calciumionen, welche fortwährend als Calciumoxalat-Kristalle aus der Lösung ausfallen, quantitativ erfaßt. Als Vergleich dienen das Kristallwachstum, welches bei Verwendung einer inhibitorfreien calciumhaltigen Standardlösung anstelle von Urin erfolgt, sowie das maximale Wachstum bei Erreichen des Löslichkeitsproduktes $\left[\mathrm{Ca}^{++}\right] \times\left[(\mathrm{COO})_{2}^{2-}\right]$. Nach Meyer \& Smith (17) enthält die metastabile Lösung je $0,44 \mathrm{mmol} / 1$ an $\mathrm{Ca}^{++}$and Oxalat. Sie wird nach Robertson (7) mit Na-Cacodylat gepuffert und mittels $\mathrm{HCl}$ auf $\mathrm{pH}$ 6,0 eingestellt.

Da nach Zugabe einer Ca-haltigen Urinprobe bzw. von inhibitorfreier Standardlösung der metastabile Bereich verlassen würde, geht man primär von einer Ca-freien Stammlösung aus. Nach $\mathrm{Zu}$ gabe einer Urinprobe bestimmt man den $\mathrm{Ca}^{++}$-Gehalt des Ansatzes und ergänzt durch eine geringe Menge einer an $\mathrm{Ca}^{++}$hochkonzentrierten $\mathrm{NaCl}$-Lösung auf den Sollwert von $0,44 \mathrm{mmol} / \mathrm{l}$. Somit wird das Kristallwachstumsexperiment unabhängig vom Ca-Gehalt des zugesetzten Urins, und der metastabile Konzentrationsbereich im Versuchsansatz - bei konstant gehaltener Calcium-Konzentration und nur minimen Schwankungen des Oxalats - gar nie verlassen.

1) Ethan-1-hydroxy-1,1-diphosphonat, EHDP, Procter \& Gamble.
Material

Chemische Substanzen Grade p.a.

$\mathrm{NaCl}$ (Fabrikat Rheinsalinen)

$\mathrm{CaCl}_{2}$ geschmolzen granuliert (Merck Art. 2083)

$\mathrm{K}_{2} \mathrm{C}_{2} \mathrm{O}_{4} \times \mathrm{H}_{2} \mathrm{O}$ (Merck Art. 5073)

Natrium-Cacodylat: $\mathrm{C}_{2} \mathrm{H}_{6} \mathrm{As}_{5} \mathrm{NaO}_{2} \times 3 \mathrm{H}_{2} \mathrm{O}$ (Dres. Bender \& Hobein AG Nr. 78-335)

Ethane-l-hydroxy-1,1-bisphosphonat (EHDP, Procter and Gamble)

Chondroitinsulfat $C$ sodium salt (from Shark Cartilage) (Calbiochem, Lot 700039)

\section{Versuchslösungen}

Alle Lösungen werden mit bidest. Wasser hergestellt. Die $[\mathrm{NaC}]$ beträgt $0,15 \mathrm{~mol} / \mathrm{l}$. Chemische Substanzen werden mit der Mettler Analy senwaage H 54 eingewogen.

a) Calciumfreie Stammlösung: In nachstehender Reihenfolge eingewogen und sogleich in bidest. Wasser gelöst.

$150 \mathrm{mmol} \mathrm{NaCl}$

$0,46 \mathrm{mmol} \mathrm{K}_{2} \mathrm{C}_{2} \mathrm{O}_{4} \times \mathrm{H}_{2} \mathrm{O}$

$40 \mathrm{mmol} \mathrm{C}_{2} \mathrm{H}_{6} \mathrm{As}_{5} \mathrm{NaO}_{2} \times 3 \mathrm{H}_{2} \mathrm{O}$

ca. 30 Tropfen $12 \mathrm{~mol} / 1 \mathrm{HCl}$ (bis $\mathrm{pH}=6,0$ )

ad $1000 \mathrm{ml}$

Diese Lösung kann bei $37^{\circ} \mathrm{C}$ maximal 3 Wochen aufbewahrt werden.

b) Inhibitorfreie Standardlösung:

$150 \mathrm{mmol} \mathrm{NaCl}$

$2 \mathrm{mmol} \mathrm{CaCl}$

ad $1000 \mathrm{ml}$

Bei $37^{\circ} \mathrm{C}$ unbeschränkt haltbar.

c) $\mathrm{Ca}^{++}$-hochkonzentrierte Lösung:

$150 \mathrm{mmol} \mathrm{NaCl}$

$15 \mathrm{mmol} \mathrm{CaCl}$

ad $1000 \mathrm{ml}$

Bei $37^{\circ} \mathrm{C}$ etwa 4 Monate haltbar.

d) Ethan-1-hydroxy-1,1-bisphosphonat-Lösung:

$0,8 \mathrm{mmol}$

ad $10 \mathrm{ml}$

e) Heparin-Lösung:

Liquémine ${ }^{\circledR}$ Roche, $1 \mathrm{ml}=5000 \mathrm{IE}$ Heparin

f) Chondroitinsulfat-Lösung:

$0,002 \mathrm{mmol}$ Chondroitinsulfat $\mathrm{C}$

ad $100 \mathrm{ml}$

Calciumoxalat-Kristallsuspension (,seed-crystals")

Die Kristalle werden nach Meyer \& Smith (12) hergestellt und jeweils 24 Stunden im Vakuum-Exsikkator über Kieselgel getrocknet, bevor je $6,0 \mathrm{mg}$ mit $0,5 \mathrm{ml}$ bidest. Wasser in Milian-Mikroröhrchen mit Deckel abgewogen, kurz auf dem Vortex geschüttelt und danach mindestens 2 Wochen bei $37^{\circ} \mathrm{C}$ gelagert werden.

Geräte

Wasserbad und Magnetrührer:

Alle Experimente werden im $37^{\circ} \mathrm{C}$-Wasserbad, welches auf 4 Magnetrührern mit konstanter Rührgeschwindigkeit von etwa $200 /$ min steht, durchgeführt.

Marius-Titrator und Zentrifuge:

In den in der Heraeus-Minifuge zentrifugierten Proben wird $\left[\mathrm{Ca}^{++}\right]$mit dem Marius-Titrator bestimmt (Titration mit Ethylenglycol-tetraessigsäure (EGTA), Calcein als fluoreszierender Indikator-Farbstoff).

pH-Meter:

Instrumentation Laboratory 228.

Osmometer:

Knauer. 。

Automatische Pipetten:

micro/pettor (smi) à 10, 20,50, 100 und $200 \mu \mathrm{l}$

Gilson „pipetman"

Papierfilter:

Schleicher \& Schüll Nr. $589^{2}$ (Porengröße etwa $\overrightarrow{7} \mu \ddot{m}$ ) 


\section{Methodik}

In einem $100 \mathrm{ml}$-Erlenmeyer, der im $37^{\circ} \mathrm{C}$-Wasserbad stcht und in dem dauernd mit etwa $200 \mathrm{U} / \mathrm{min}$ gerührt wird, stellt man einen Versuchsansatz für cin Calciumoxalat-Wachstumsexperiment wie folgt zusammen:

\section{$95,0 \mathrm{ml}$ Stammlösung (Calcium-frei)}

$3,0 \mathrm{ml}$ inhibitorfreie Standardlösung bzw. Spontanurin

$x \mathrm{ml}$ hochkonzentrierte $\mathrm{Ca}^{++}$-Lösung

Dabci ist $x$ abhängig von der $\left[\mathrm{Ca}^{++}\right]$sowohl der Standardlösung bzw. Urinprobe als auch der hochkonzentrierten $\mathrm{Ca}^{++}$-Lösung selbst. Beide Konzentrationen werden vor Beginn cihes Experimentes bestimmt, worauf im Versuclisansatz durch entsprechende Zugabe hoclikonzentrierter $\mathrm{Ca}^{++}{ }^{+}$-Lösung der. $\left[\mathrm{Ca}^{++}\right]$-Sollwert von $0,44 \mathrm{mmol} / \mathrm{l}$ eingestellt wird. Anschließend gibt man $0,5 \mathrm{ml}$ Calciumoxalat-Kristallsuspension $\mathrm{zu}$, was den Beginn der Wachstumsphase $(t=0)$ darstcllt. Nach 3,10 und 60 Minuten entnimmt man dem Ansatz $3,0 \mathrm{ml}$ Suspension und zentrifugiert diese während 4 Minuten mit $5000 \mathrm{U} / \mathrm{min}$ bis zur Sedimentation der Kristallaggregate (Raumtemperatur). Aus dem Uberstand werden $2 \mathrm{mal}$ (Doppelwerte) je $200 \mu \mathrm{l}$ entnommen, in denen $\left[\mathrm{Ca}^{++}\right]$mittels Titration mit Ethylenglycol-tctracssigsäure (Calcein als fluoreszicrender Indikator-Farbstoff) gemessen wird.

Das Standardexpcriment mit inhibitorfreicr Standardlösung $(30 \mathrm{ml} / \mathrm{l}$ ) wurde über Monate hinweg regelmäßig wiederholt, um die Reproduzierbarkeit der Methode zu überprüfen.

Mit diesem Versuchsansatz wurden zunächst bekannte Inhibitoren in vitro getestet, indem unmittelbar vor Zugabe der Calciumoxalat-Kristallsuspension kleine Mengen hochkonzentrierter Inhibitorenlösungen beigegeben wurden. Einerseits handelte es sich - wic bei Robertson et al. (9) sowie Pak et al. (10) ähnlich beschrieben - um Ethan-1-hydroxy-1,1-bisphosphonat $(2,4$ und $8 \mu \mathrm{mol} / \mathrm{l})$, andererseits um Heparin $(0,23,1,13$ und $5,66 \mu \mathrm{mol} / \mathrm{l})$ und Chondroitinsulfat $\mathrm{C}(10,50$ und $250 \mathrm{nmol} / \mathrm{l})$.

Die Bestimmung der Hemmkraft von Urin erfolgt analog, indem statt inhibitorfreier Standardlösung Urin $(30 \mathrm{ml} / \mathrm{l})$ zugesetzt wird. Zur Untersuchung gelangen Spontanurine nüch terner und stark hydrierter Probanden. Die Spontanurine werden bis zur Verarbcitung unbehandelt in Plastikbehältern aufbewahrt und tiefgefroren. Vor der Verwendung in Calciumoxalat-Wachstumsexperimenten wird der Urin im $37^{\circ} \mathrm{C}$-Wärmeschrank aufgetaut und anschließend mittels Papierfilter filtriert. Im Filtrat wird die Osmolarität bestimmt. Beträgt sic über $300 \mathrm{mosmol} / \mathrm{l}$, wird der Urin mit bidest. Wasser auf diesen Wert vcrdünnt. So crhält man vergleichbare Mengen gelöster Urinbestandteile und befindet sich zudem im Bereich der Osmolarität physiologischer $\mathrm{NaCl}$-Lösung. Vorversuche ergaben, daß dic Hemmkraft eines Urins unterhalb $500 \mathrm{mosmol} / 1 \mathrm{mit}$ zunehmender Verdünnung so wenig abnimmt, daß mit der Genauigkeit unserer Meßmethodik keine signifikanten Unterschiede 'mehr festgestellt werden können. Entscheidender ist noch, daß durch die Verwendung verdünnter Urine - bei konstant gehaltener $\mathrm{Ca}^{++}-$Konzentration - die Schwankungen der Oxalatkonzentration im Versuchsansatz so minim bleiben, daß der metastabile Bereich bezüglich des Produktes $\left[\mathrm{Ca}^{++}\right] \mathrm{X}$ [(COO) $\left.{ }_{2}^{2-}\right]$ nie verlassen werden kann, was sich auch anhand der Nomogramme von Marshall \& Robertson (13) zeigen läßt.

24 Normalpersonen wurden untersucht. Es handelte sich um regelmäßige Blutspender aus dem Blutspendezentrum Limmattal SRK in Schlieren ZH (wir danken dem Leiter, Herrn Dr. M. Frey. Wettstein, für die Mithilfe) sowic um Mitarbeiter der Nephrologischen Station des Departementes für Innere Medizin am Universitätsspital Zürich. Sie hatten alle zum Zeitpunkt der Untersuchung bezüglich Nephrolithiasis eine stumme Anamnese und waren ohne manifesten Harnwegsinfekt.

Die verwendeten ,seed-crystals" wurden sowohl vor als auch nach der Verwendung in Kristallwachstumsexperimenten mehrmals mittels Röntgen-Diffraktion untersucht (unter freundlicher Mithilfe von Herrn Dr. Asper, Med. Chem. Zentrallaboratorium des Universitätsspitals Zürich). Es handelte sich dabei um reine Calciumoxalat-Monohydrat-Kristalle.

Die Resultate aller Experimente wurden graphisch dargestellt, indem die relative Abnahme $\left[\mathrm{Ca}^{++}\right]$zur Zeit $t\left(\Delta \mathrm{Ca}_{t}\right)$ auf der Ordinate gegen die Zcit $(t)$ aufgetragen wurde. Dic entstchende hyperbolische Kurve kann nach Bijvoet ct al. (14) durch 2 Para- meter beschrieben werden: Einerseits durch eine Asymptote, welche der maximalen Abnahme an $\left[\mathrm{Ca}^{++}\right]$zur Zeit $\mathrm{t}=\infty$ entspricht $(\Delta \mathrm{Ca})$, andererseits durch die Zeit $\mathrm{T}_{\mathrm{m}}, \mathrm{zu}$ der die Abnahme an $\left[\mathrm{Ca}^{++}\right]$halbmaximal ist:

$$
\Delta \mathrm{Ca}_{\mathrm{t}} / \Delta \mathrm{Ca} \infty=\mathrm{t} /\left(\mathrm{T}_{\mathrm{m}}+\mathrm{t}\right)
$$

Durch Umformung ergibt sich

$$
t / \Delta C_{a_{t}}=T_{m} / \Delta C a \infty+(1 / \Delta C a \infty) t
$$

und durch Auflösung nach $\mathrm{T}_{\mathrm{m}}$

$$
T_{m}=\left(\Delta C a \infty / \Delta C a_{t}\right) t-t
$$

$\Delta \mathrm{Ca}_{\mathrm{t}}$, dic prozentuale Abnahme der $\left[\mathrm{Ca}^{++}\right]$zur Zcit t, ergibt sich aus der Formel

$$
\mathrm{Ca}_{\mathrm{t}}=100 \%-\left(\left[\mathrm{Ca}^{++} \mid \mathrm{t} /\left[\mathrm{Ca}^{++}\right]_{0}\right) \times 100 \%\right.
$$

und wird experimentell bestimmt. $\left[\mathrm{Ca}^{++}\right]_{0}$ (Ausgangswert) beträgt $0,44 \mathrm{mmol} / \mathrm{l}, \Delta \mathrm{Ca} \infty$ wurde bestimmt, indem das Standardexperiment inehrmals über 24 Stunden verlängert wurde. Nach dieser Zcit wird ein Gleichgewichtszustand erreicht, bei dem pro Zeitcinheit glcichvielc Calcium- und Oxalationen in Lösung gehen wie solche als Calciumoxalat-Kristalle aus der Lösung ausfallen. Der Mittelwert aus 5 solchen Standardexperimenten betrug $37,38 \%$.

Es wurde nun für jeden einzelnen Versuchsansatz $T_{m}$ basierend auf $\Delta \mathrm{Ca}_{60}$ errechnet, d.h. dic Zcit, in der in cinem bestimmten Ansatz dic Hälf te des maximal erreichbaren $\left[\mathrm{Ca}^{++} \mid\right.$-Abfalles crfolgt ist. $T_{m}$ ist eine charaktcristische Größe für die globale Hemmkraft eines einzelnen Versuchansatzes, da sie die Geschwindigkeit, mit der sich der hyperbolische Kurvenverlauf des Kristallwachstums pro Zeiteinheit der Asymptoten $\Delta \mathrm{Ca}_{60}$ nähert, ausdrückt.

\section{Resultate}

Die Reproduzierbarkeit der dargelegten Methode ist, wie Tabelle 1 zeigt, mit einem Variationskoeffizienten von $4,6 \%$ gut für die Messungen der relativen $\left[\mathrm{Ca}^{++}\right]-\mathrm{Ab}$ nahme nach 60 Minuten $\left(\Delta \mathrm{Ca}_{60}\right)$, hingegen ungenügend für Messungen nach bereits 3 Minuten, wo die $\left[\mathrm{Ca}^{++}\right] /$ Zeit-Kurve steiler verläuft.

In Abbildung 1 sind in semilogarithmischer Darstellung Hemmkraft (ausgedrückt durch die Größe $T_{m}$ ) gegen Konzentration dreier bekannter Inhibitoren im Versuchsansatz aufgetragen (Dosis-Wirkungs-Geraden). Eth an-1hydroxy-1,1-bisphosphonat ist der stärkste Hemmer; es läßt sich mit der kleinsten Dosissteigerung der größte Zuwachs an Hemmkraft erzielen.

In einem Vorversuch wurde der Effekt der Verdünnung eines Spontanurins mit bidest. Wasser untersucht. Der unverdünnte Urin (1200 mosmol/l) ergab im Kristallwachstumsexperiment einen $\Delta \mathrm{Ca}_{60}$-Wert von $20,1 \%$. Die entsprechenden Werte für die Verdünnungen auf 700 , 500 und 300 mosmol/l betrugen $23,5 \%, 24,2 \%$ und $24,7 \%$. Der Unterschied zwischen den 2 letztgenannten Ergebnissen ist nicht mehr signifikant.

$\mathrm{Da}\left[\mathrm{Ca}^{++}\right]$im Versuchsansatz konstant gehal ten wird, interessierten die Abweichungen der [(COO $\left.)_{2}^{2-}\right]$ vom Ausgangswert im Standardansatz $(0,440 \mathrm{mmol} / \mathrm{l}=$ $38,50 \mathrm{mg} / \mathrm{l} \mathrm{Kalium}-O x a l a t)$, nachdem Urin $(30 \mathrm{ml} / \mathrm{l}) \mathrm{zu}$ gesetzt worden waren. Die mittlere Ausgangskonzcntration an Oxalat in 7 Versuchsansätzen betrug nach dem 


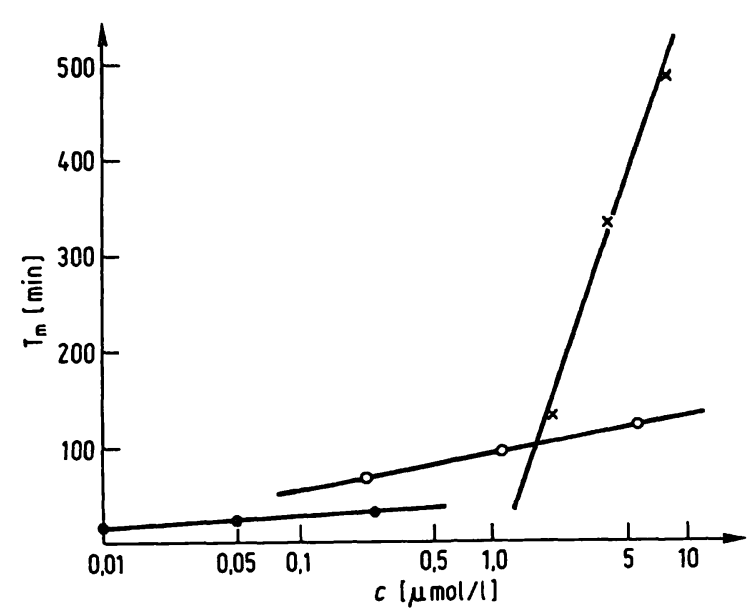

Abb. 1. Semilogarithmische Darstellung der Beziehung $T_{m} /$ Konzentration des Inhibitors (Dosis-Wirkungs-Geraden) für Ethan-1-hydroxy-1,1-bisphosphonat $(x-x)$, Heparin $(0-0)$ und Chondroitinsulfat $C(\bullet-\bullet)$ in je 3 verschiedenen Konzentrationen.

Zusatz 7 verschiedener Urine $0,443 \mathrm{mmol} / 1$. Bei diesen minimalen Schwankungen der Oxalat-Konzentrationen und konstant gehaltenem Calcium konnte somit anhand der von Marshall \& Robertson übernommenen Nomogramme (13) gezeigt werden, daß der metastabile Bereich bezüglich des Produkts $\left[\mathrm{Ca}^{++}\right] \times\left[(\mathrm{COO})_{2}^{2-}\right]$ nie verlassen wird, solange verdünnte Urine verwendet werden.

Die Reproduzierbarkeit der Methode nach wiederholter Zugabe des gleichen, gut hemmenden Urins war mit einem Variationskoeffizienten von $6,8 \%$ für die $\left[\mathrm{Ca}^{++}\right]$Abnahme nach 60 Minuten $(n=6)$ annähernd gleich gut wie im Standardexperiment ohne Urin, hingegen für Messungen nach bereits 3 Minuten (VK $=69,46 \%$ ) noch viel unzuverlässiger als bereits im Standardversuch (vgl. Tab. 1).

Bei den 24 untersuchten Spontanurinen von Normalpersonen betragen die Mittelwerte für $\Delta \mathrm{Ca}_{60} 26,96 \pm 2,61 \%$ $(\bar{x} \pm s)$ und für $T_{m} 23,82 \pm 8,99 \min (\bar{x} \pm s)$. Demzufolge resultiert durch die Verdünnung eine nicht sehr massive, aber gegenüber dem Standardexperiment ohne Urin noch signifikant bessere Hemmung des Kristallwachstums.

Tab. 1. Mittelwert ( $\overline{\mathbf{x}})$, Standardabweichung (S.D.), Standardfehler (S.E.M.) und Variationskoeffizient (VK) der relativen $\mathrm{Abnahme} \mathrm{der} \mathrm{Calciumkonzentration}\left(\Delta \mathrm{Ca}_{\mathrm{t}}\right)$ nach 3, 10 und 60 Minuten sowie der Zeit der halbmaximalen Calciumabnahme $\left(T_{m}\right)$ in 23 Standardexperimenten, durchgefuhrt in einem Zeitraum von 10 Monaten.

\begin{tabular}{lllll}
\hline $\mathrm{n}=23$ & $\begin{array}{l}\Delta \mathrm{Ca}_{3} \\
(\%)\end{array}$ & \multicolumn{1}{c}{$\begin{array}{l}\Delta \mathrm{Ca}_{10} \\
(\%)\end{array}$} & \multicolumn{1}{c}{$\begin{array}{l}\Delta \mathrm{Ca}_{60} \\
(\%)\end{array}$} & \multicolumn{1}{c}{\begin{tabular}{l}
\multicolumn{1}{c}{$\mathrm{T}_{\mathrm{m}}$} \\
$(\mathrm{min})$
\end{tabular}} \\
\hline$\overline{\mathrm{x}}$ & 7,230 & 13,935 & 29,100 & 17,230 \\
S.D. & 1,106 & 0,885 & 1,348 & 3,548 \\
S.E.M. & 0,231 & 0,184 & 0,281 & 0,740 \\
VK & 15,3 & 6,4 & 4,6 & 20,8 \\
\hline
\end{tabular}

\section{Diskussion}

Unsere nach Meyer \& Smith (12) vereinfachte Methode, mit der wir unter Ausschluß der Probleme betreffend die Ubersättigung an kristallbildenden Ionen ausschließlich die Hemmung des Calciumoxalat-Kristallwachstums in vitro untersuchten, erwies sich als technisch einfach und gut reproduzierbar, vor allem für Messungen der relativen $\left[\mathrm{Ca}^{++}\right]$-Abnahme (als Ausdruck der Hemmkraft) $60 \mathrm{Mi}$ nuten nach Beginn eines Kristallwachstumsexperimentes. Dies gilt sowohl für Experimente mit inhibitorfreier Standardlösung als auch für solche mit Spontanurinproben. Zuverlässige Messungen im Bereich des sehr raschen Kristallwachstums zu Beginn des Experiments sind hingegen nicht möglich. Die Resultate aus 23 Standardversuchen lassen sich in etwa mit denjenigen vergleichen, die Meyer \& Smith aus 3 solchen Experimenten graphisch darstellten (15). Es läßt sich dort ein Abfall der Ausgangskonzentration an $\mathrm{Ca}^{++}$von etwa $16 \%$ nach 10 Minuten und von etwa $35 \%$ nach 60 Minuten extrapolieren. Vergleiche mit Resultaten aus den Arbeiten der Gruppe um Robertson sind nicht möglich, da dort das CalciumoxalatKristallwachstum anhand der Zunahme von Aggregaten einer bestimmten Größe im Lauf der Zeit gemessen wird. Direkte Vergleiche mit den Resultaten von Bijvoet, der den Einbau radioaktiv markierten Calciums in Calciumoxalat-Kristalle mißt (14), sind ebenfalls unmöglich; dagegen liefert die dort dargestellte mathematische Auswertung der Ergebnisse mit $T_{m}$ eine Größe, welche auch in unseren Versuchen den ganzen hyperbolischen Verlauf der $\left[\mathrm{Ca}^{++}\right]$-Abnahme pro Zeiteinheit zu charakterisieren vermag.

Ethan-1-hydroxy-1,1-bisphosphonat und Heparin zeigten in unserem Standardversuch die bekannte, dosisabhängige Hemmwirkung auf das Calciumoxalat-Kristallwachstum in vitro. Bei ähnlichen Konzentrationen im Versuchsansatz hemmte Ethan-1-hydroxy-1,1-bisphosphonat stärker bzw. ließ sich durch eine vergleichsweise eher kleinere Dosissteigerung bei Ethan-1-hydroxy-1,1-bisphosphonat eine bedeutend stärkere Hemmungsverbesserung erzielen als mit Heparin. Chondroitinsulfat $C$ erwies sich mit unserer Meßmethodik als schwacher Inhibitor des Calciumoxalat-Kristallwachstums, dies im Gegensatz zu Ligabue et al., welche mit ihrer ${ }^{14} \mathrm{C}$-markiertes Oxalat messenden Technik zeigten, daß Chondroitinsulfat $C$ in noch viel geringerer Konzentration als in unseren Versuchen ein sehr guter Hemmer des Calciumoxalat-Kristallwachstums ist (16).

Die Verwendung verdünnter Urine im Kristallwachstumsexperiment hat den großen Vorteil, daß eine Ubersättigung an kristallbildenden Ionen praktisch ausgeschlossen ist. Dafür ist die Konzentration an Hemmsubstanżen geringer, so daß̧ Unterschiede in der Hemmkraft einzelner Urine weniger deutlich werden. Es erscheint vorteilhäft, die Urine stark hydrierter Probaninden zu untersuchen und sie nur nach Bedarf wenig nachżuverdünnen, da die Verdünnung auf diese Art ,physiologischer" ist: alleiniges Nachverdünnen gelöster Urine soll möglicherweise, wie 
Leskovar in seiner Ubersichtsarbeit ausfuhrt (3), ein verändertes Verhalten der Inhibitoren nach sich ziehen. Das Einfrieren der frischen Urinproben ohne spezielle Vorbehandlung wurde von Meyer \& Smith ebenfalls angewandt (17).

\section{Literatur}

1. Williams, H. E. (1974), New. Engl. J. Med. 290, 33-38.

2. Fleisch, H. (1974), Nieren- und Hochdruckkrankheiten 3, 112-115.

3. Leskovar, P. (1979), Aktuelle Nephrologie, Heft 1, Wissenschaftliche Information, Fresenius-Stiftung, 149-175.

4. Robertson, W. G., Peacock, M., Marshall, R. W., Marshall, D. H. \& Nordin, B. E. C. (1976), New Engl. J. Med. 294, 249-252.

5. Thomas, W. C. \& Howard, J. E. (1959), Trans. Ass. Am. Physicians 72, 181-187.

6. Robertson, W. G. \& Peacock, M. (1972), Clin. Sci. 43, 499506.

7. Smith, L. H., Meyer, J. L. \& McCall, J. T. (1973), Int. Symp. Renal Stone Res. 1972, Madrid (Cifuentes Delatte, ed.) pp. 318-327, Karger, Basel.

8. Meyer, J. L. \& Smith, L. H. (1975), Invest. Urol. 13, 36-39.

\section{Verdankungen}

Die technische Assistenz von Frau U. Flueler, Frau A. Meier und Frau M. Stillhart-Huggler wird bestens verdankt. Frau B. Rutz leistete zuverlässige und exakte Schreibarbeit.
9. Robertson, W. G., Peacock, M. \& Nordin, B. E. C. (1972), Clin. Chim. Acta 43, 31-37.

10. Pak, C. Y. C., Ohata, M. \& Holz, K. (1975), Kidney Int. 7, $154-160$.

11. Robertson, W. G., Peacock, M., Marshall, R. W. \& Knowles, F. (1974), Clin. Sci. Mol. Med. 47, 13-22.

12. Meyer, J. L. \& Smith, L. H. (1975), Invest. Urol. 13, 31-35.

13. Marshall, R. W. \& Robertson, W. G. (1976), Clin. Chim. Acta 72, 253-260.

14. Bijvoet, O. L. M., van der Linden, H. \& Will, E. J. (1978), Urol. Nephrol. 11, Pathogenese und Klinik der Harnsteine VI, 224-232. (Verlag Steinkopff, Darmstadt).

15. Smith, L. H., Werness, P. G., Bergert, J. J. \& Lee, K. E. (1978), Kidney Int. 14, 663.

16. Ligabue, E., Fini, M. \& Robertson, W. G. (1979), Clin. Chim. Acta 98, 39-46.

PD Dr. U. Binswanger Medizinische Klinik Nephrologische Station Universitätsspital Rämistraße 100 CH-8091 Zürich 
